\title{
Multilocus dataset reveals demographic histories of two peat mosses in Europe
}

\author{
Péter Szövényi*1,2, Zsófia Hock1,2, Jakob J Schneller ${ }^{1}$ and Zoltán Tóth²
}

Address: ${ }^{1}$ Institute of Systematic Botany, University of Zurich, Zurich, 8008, Zollikerstrasse 107, Switzerland and ${ }^{2}$ Department of Plant Taxonomy and Ecology, Eötvös Loránd University, Budapest, 1117, Pázmány Péter sétány 1/C, Hungary

Email: Péter Szövényi* - szovenyi@systbot.uzh.ch; Zsófia Hock - hockzsofia@hotmail.com; Jakob J Schneller - schnell@systbot.uzh.ch; Zoltán Tóth - tothz9@ramet.elte.hu

* Corresponding author

Published: 22 August 2007

BMC Evolutionary Biology 2007, 7:144 doi:10.1 I86/147|-2|48-7-144
Received: 22 December 2006

Accepted: 22 August 2007

This article is available from: http://www.biomedcentral.com/|47|-2/48/7//44

(C) 2007 Szövényi et al; licensee BioMed Central Ltd.

This is an Open Access article distributed under the terms of the Creative Commons Attribution License (http://creativecommons.org/licenses/by/2.0), which permits unrestricted use, distribution, and reproduction in any medium, provided the original work is properly cited.

\begin{abstract}
Background: Revealing the past and present demographic history of populations is of high importance to evaluate the conservation status of species. Demographic data can be obtained by direct monitoring or by analysing data of historical and recent collections. Although these methods provide the most detailed information they are very time consuming. Another alternative way is to make use of the information accumulated in the species' DNA over its history. Recent development of the coalescent theory makes it possible to reconstruct the demographic history of species using nucleotide polymorphism data. To separate the effect of natural selection and demography, multilocus analysis is needed because these two forces can produce similar patterns of polymorphisms. In this study we investigated the amount and pattern of sequence variability of a Europe wide sample set of two peat moss species (Sphagnum fimbriatum and S. squarrosum) with similar distributions and mating systems but presumably contrasting historical demographies using 3 regions of the nuclear genome (appr. 3000 bps). We aimed to draw inferences concerning demographic, and phylogeographic histories of the species.
\end{abstract}

Results: All three nuclear regions supported the presence of an Atlantic and Non-Atlantic clade of $S$. fimbriatum suggesting glacial survival of the species along the Atlantic coast of Europe. Contrarily, S. squarrosum haplotypes showed three clades but no geographic structure at all. Maximum likelihood, mismatch and Bayesian analyses supported a severe historical bottleneck and a relatively recent demographic expansion of the Non-Atlantic clade of $S$. fimbriatum, whereas size of $S$. squarrosum populations has probably decreased in the past. Species wide molecular diversity of the two species was nearly the same with an excess of replacement mutations in S. fimbriatum. Similar levels of molecular diversity, contrasting phylogeographic patterns and excess of replacement mutations in $S$. fimbriatum compared to $S$. squarrosum mirror unexpected differences in the demography and population history of the species.

Conclusion: This study represents the first detailed European wide phylodemographic investigation on bryophytes and shows how pattern of nucleotide polymorphism can reveal unexpected differences in the population history of haploid plants with seemingly similar characteristics. 


\section{Background}

Geographic distribution of species is continuously shaped by several extrinsic factors causing repeated historical range and demographic expansions/contractions $[1,2]$. Using historical observations, short-term range expansions and contractions, together with associated demographic changes, have been documented in several, mostly invasive species in Europe and also world-wide [36]. From a conservation biological point of view, it is of high importance to reconstruct the historical demography of populations, which can help to work out conservation strategies. In addition, correlations between demographic changes and climatic oscillations may be useful to predict species responses to the accelerated climate change [7]. If a series of temporarily spaced data is available, demographic changes over several decades are relatively easy to reconstruct. However, comparison of historical and recent observations is challenging, among others because of the difficulties emerging when comparing data gathered by different sampling strategies [8].

Methods using the current level and distribution of genetic variability to estimate the historical demography of populations provide an additional tool to test demographic hypotheses suggested by simple historical observations [9-12]. Historical demography considerably influences both qualitative and quantitative properties of sequence-level polymorphisms at neutrally evolving sites $[13,14]$. Consequently, based on the current pattern of sequence-level polymorphisms, it is possible to distinguish among populations, which currently underwent a demographic expansion and those, which are in a declining phase. Populations after a demographic expansion are described by rapid coalescent events following long branches on the coalescent tree [14]. In the mismatch distribution, long branches lead to an excess of low frequency polymorphisms $[15,16]$.

Unfortunately, selective forces might lead to the same pattern of molecular variation as would be expected in an expanding population. Investigating noncoding, neutrally evolving sequence portions does not overcome the problem of selection because they might be influenced by hitchhiking effects of linked loci [17]. Although selective forces and demography might produce the same pattern of polymorphism, selection affects the genome locally, whereas demography extends its effect on the whole genome. Consequently, investigating multiple, unlinked loci can help to separate the influence of selection and demography on the pattern of nucleotide polymorphism $[18,19]$.

In the present study, we aimed to reconstruct the demographic histories of two peat moss species based on nucleotide polymorphism data. Peat mosses are an ancient plant group with a worldwide distribution [20] and represent a model system for population genetic research in bryophytes [21-24]. In addition, phylogenetically closely related species pairs with similar distribution patterns and life history characteristics provide an outstanding opportunity to test the influence of species-specific demography on nucleotide polymorphism.

To concentrate on the effect of demography and minimize the influence of other possible factors on the pattern and amount of sequence-level variability, two phylogenetically closely related species with similar distributions, ecological requirements and mating systems have been selected. Both species are monoecious (produce antheridia and archegonia on the same gametophore) and distributed from the Iberian Peninsula to Svalbard across Europe. They frequently occur together at the edge of mires under Alnus or Salix stands and can tolerate higher nutrient concentrations $[20,25]$. Although they share similar characteristics, recent investigations show that $S$. fimbriatum is more successful in colonizing open soil surfaces and produces more rapidly sporophytes after establishment than S. squarrosum [26]. In addition, analyses of past and current distributions of the species show that $S$. fimbriatum is presumably experiencing a rapid expansion at least in some parts of Europe, whereas $S$. squarrosum shows no evidence of a recent population demographic change [27].

Previous investigations on a limited number of accessions using chloroplast sequences, suggested contrasting phylogeographic patterns in the monoecious $S$. fimbriatum and S. squarrosum in Europe [28]. Although chloroplast markers allowed drawing of phylogeographic hypotheses, very low resolution of the dataset hampered any further statistical inference. Moreover, the chloroplast genome consists of completely linked loci and thus may be strongly influenced by selective forces. Consequently, it is impossible to separate the effect of demography and natural selection on nucleotide polymorphisms using only chloroplast sequence data.

Therefore, this study aims to investigate the historical demography of the two species in detail using more detailed sampling, and sequence data of three unlinked nuclear regions covering appr. 3000 bps of the genome. We intended to differentiate between selective and demographic forces and were particularly interested in the following questions: 1 . Does phylogeographic structure depend on the loci investigated? 2. Does multilocus analysis support assumed population expansion of S. fimbriatum? 3. What kind of demographic histories could lead to the current amount and pattern of molecular variability? 


\section{Results Geographic distribution of haplotypes}

In $S$. fimbriatum, the GapC and the RAPDa regions resolved the highest number of haplotypes (19 and 16 respectively), whereas the ITS region had much less resolution (9 haplotypes). All three regions supported a welldefined split of haplotypes into two groups (Figure 1). One lineage, further referred to as "Atlantic clade" occurs along the Atlantic coast of Spain, France and southern part of Britain. The rest of the accessions grouped into a clade extending from Southern France to Scandinavia ("NonAtlantic clade") with one or two frequent and several rare haplotypes. In contrast to the ITS and RAPDa regions, the GapC gene gives further interpretable resolution within the Non-Atlantic clade. Plants from eastern part of Spain (haplotypes G and H, Figure 1) form a separate, geographically well-delimited clade, also accessions from Austria and Hungary (haplotype E, except one occurrence in Scandinavia, Figure 1) show some geographic affinity.
In $S$. squarrosum, all three nuclear markers provided similar distributions of haplotypes, however, markers differed much more in their resolution than in $S$. fimbriatum (Figure 2). The ITS region contained only two singleton polymorphisms and one indel, which divided the accessions into two haplotype groups with similar numbers of accessions. The GapC gene resolved 11 haplotypes, whereas in the RAPDa region no substitutions were found except two informative indels. In spite of the lack of substitutions, the RAPDa region contained a complete dinucleotide repeat, which showed considerable variability and resolved 20 haplotypes. The dinucleotide repeat was also present in $S$. fimbriatum sequences but showed no variability. In contrast to S. fimbriatum, S. squarrosum haplotypes show no clear geographic affinity. One of the ITS types tends to be more frequent in the south than in the north, but with a considerable admixture. RAPDa haplotypes show no clear geographic pattern either. Haplotypes of the GapC gene

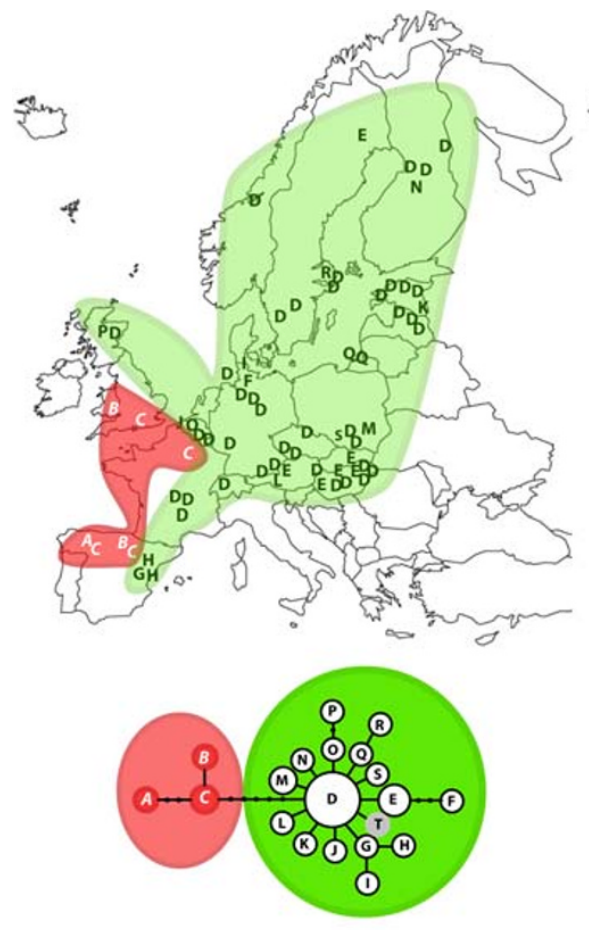

GapC

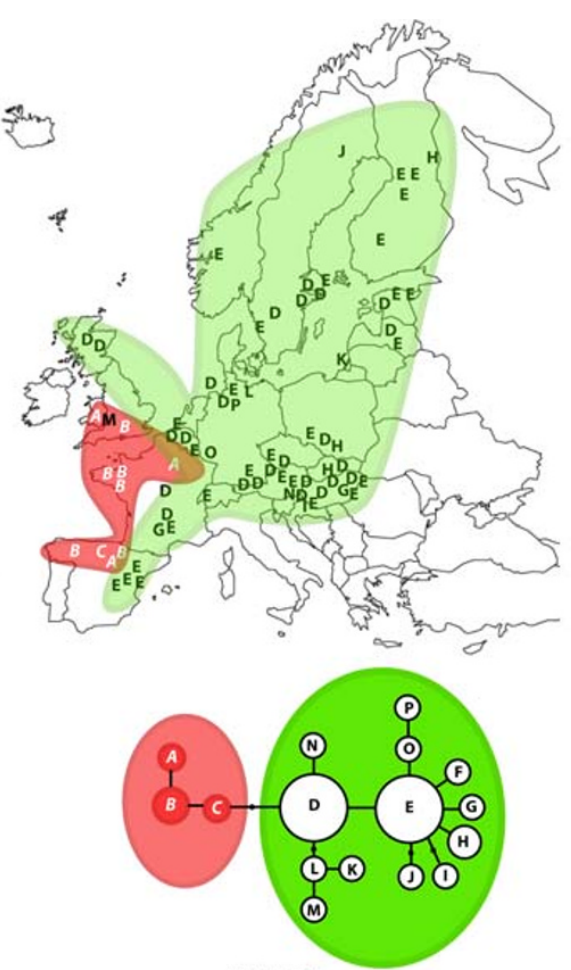

RAPDa
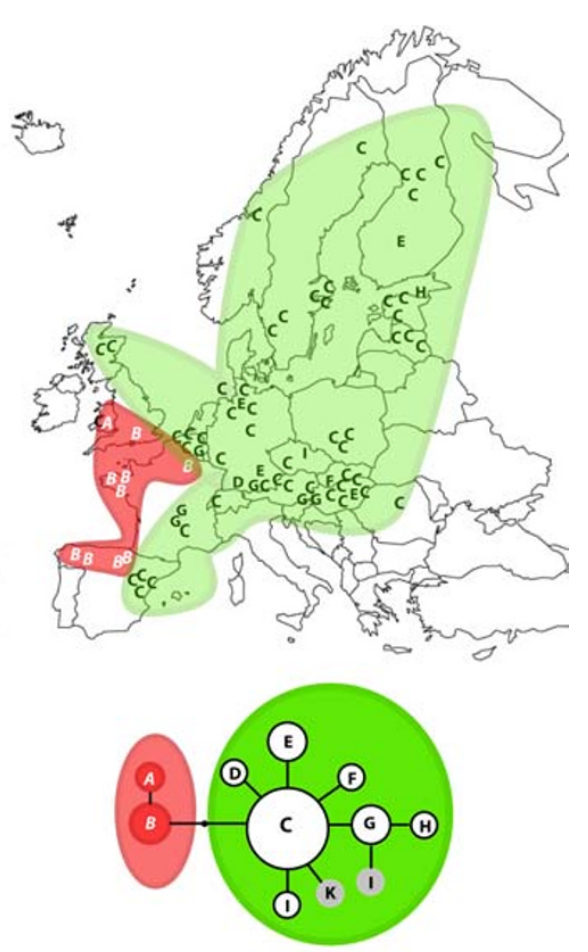

ITS

\section{Figure I}

Geographic distribution of S. fimbriatum haplotypes and the corresponding maximum parsimony networks. In each haplotype network, letters in circles represent haplotypes found. Haplotypes found only in America are shown in circles with gray background. Missing haplotypes are marked with black dots and lines connecting haplotypes denote one mutational change. The Atlantic and Non-Atlantic clades are shown within red and green ellipses respectively. 
are also widely distributed and only two of them show any geographic grouping (D, G Figure 2).

\section{Molecular polymorphism, tests of neutrality and recombination}

Estimates of $\theta$ were lower in $S$. squarrosum for the ITS region but were similar for the GapC gene in both species (Table 1). The GapC gene of S. fimbriatum showed high diversity at synonymous sites whereas in $S$. squarrosum all mutations were found in noncoding regions.

The RAPDa region has been excluded from the multilocus HKA test because it showed no point mutations in $S$. squarrosum and only the microsatellite repeat was variable. No significant deviation from the neutral model was detected using the multilocus HKA test $(\mathrm{p}=0.48)$. Despite of the excess of non-synonymous substitutions in S. fimbriatum compared to $S$. squarrosum (Table 1), the McDonald-Kreitman test (GapC gene) was not significant either $(\mathrm{p}=0.50)$. In S. squarrosum, Tajima's D and Fay and Wu's $\mathrm{H}$ statistics were never significantly different from the neutral expectations.

Using all European accessions of S. fimbriatum, Tajima's D was significant for all three nuclear regions investigated when including gaps (Table 1). However, it turned out to be non-significant when excluding gaps, except for the GapC gene. Fay and Wu's H was never significant. Analysing accessions of the Non-Atlantic clade of $S$. fimbriatum, Tajima's D was significantly negative except the RAPDa region. The latter showed a significant negative value as well when gaps were included. The Atlantic clade showed no significant deviations from neutrality.
No signs of recombination were found in RAPDa and ITS data sets of both species. GapC sequences of $S$. fimbriatum showed one incompatible pair and an $R_{m}$ value of 1 . The two accessions causing the incompatibility were removed from the data set in further analyses. No signs of recombination were found in GapC sequences of S. squarrosum.

\section{Population growth}

Without gaps, Fu's $\mathrm{F}_{\mathrm{s}}$ statistic was only significant in the case of the GapC gene including all European accessions of $S$. fimbriatum (Table 2). In contrast, when gaps were used in the calculations, it turned out to be significantly different from a constant population in all three regions investigated. Analysing only the Non-Atlantic group of accessions, test statistic was significantly negative in all three genomic regions when including gaps. None of these statistics were significant using European accessions of $S$. squarrosum and accessions of the Atlantic clade of $S$. fimbriatum.

Due to low substitutional variability, ITS and RAPDa regions of both species had to be excluded from the maximum likelihood estimation of exponential growth rate and historical theta. In S. fimbriatum estimation was made separately for all European accessions and for the NonAtlantic clade. Analysis using all European accessions of $S$. fimbriatum showed about 4 times higher growth rates than in S. squarrosum, however, values were not significantly different from a shrinking, stable or expanding population (Table 3). Historical theta values were nearly the same for both species. Estimation provided two orders of magnitude higher growth rates and about one order of magnitude higher theta estimates for the Non-Atlantic clade of $S$. fimbriatum compared to $S$. squarrosum. Growth

Table I: Nucleotide polymorphisms of the regions investigated. Number of sequences, aligned length, nucleotide diversity estimates and neutrality tests of the nuclear regions investigated. Tajima's D was calculated taking nucleotide substitutions or nucleotide substitutions and gaps into account.

\begin{tabular}{|c|c|c|c|c|c|c|c|c|c|c|c|}
\hline & \multirow[t]{2}{*}{$\mathrm{N}$} & \multirow{2}{*}{$\begin{array}{l}\text { Aligned } \\
\text { lenght } \\
\text { in bps }\end{array}$} & \multirow{2}{*}{$\theta_{\mathrm{w} \text { total }}$} & \multirow{2}{*}{$\theta_{\pi \text { total }}$} & \multirow[t]{2}{*}{$\theta_{\mathrm{w} \text { syn }}$} & \multirow[t]{2}{*}{$\theta_{\mathrm{w} \text { nonsyn }}$} & \multirow[t]{2}{*}{$\theta_{\mathrm{w} \text { sil }}$} & \multicolumn{3}{|c|}{ Tajima's D (with gaps/without gaps) } & \multirow{2}{*}{$\begin{array}{l}\text { Fay and } \\
\text { Wu's H } \\
\text { Total data } \\
\text { set }\end{array}$} \\
\hline & & & & & & & & Atlantic clade & Non-Atlantic clade & Total data set & \\
\hline \multicolumn{12}{|c|}{ S. fimbriatum } \\
\hline RAPDa & 80 & 993 & 0.00103 & 0.00120 & - & - & - & $-0.6909 /-1.1117$ & $-|.7448 * / 0.474|$ & $-1.3992 * / 0.3600$ & 0.4044 \\
\hline ITS & 90 & 614 & 0.00164 & 0.00077 & - & - & - & $-1.1117 /-1.1117$ & $-1.7460 * /-1.6393 *$ & $-1.5950 * /-1.1427$ & 0.4155 \\
\hline GapC & 76 & 1566 & 0.00236 & 0.00101 & 0.01124 & 0.00186 & 0.00267 & $-0.8764 /-1.3272$ & $-2.2321 * /-1.9777 *$ & $\begin{array}{l}-1.9074 * /- \\
1.8090 *\end{array}$ & -3.2821 \\
\hline \multicolumn{12}{|c|}{ S. squarrosum } \\
\hline RAPDa & 57 & 1033 & - & - & - & - & - & - & - & - & - \\
\hline ITS & 60 & 668 & 0.00033 & 0.00005 & - & - & - & - & - & $-0.2501 /-1.0857$ & 0.0655 \\
\hline GapC & 50 & $156 \mid$ & 0.00201 & 0.00202 & 0.00000 & 0.00000 & 0.00201 & - & - & $-0.4287 / 0.0279$ & -1.2114 \\
\hline
\end{tabular}

$\theta_{\mathrm{w} \text { total }}:$ Watterson's estimator calculated using all accessions; $\theta_{\pi \text { total }}$ : Tajima's estimator calculated using all accessions; syn: synonymous sites; nonsyn: nonsynonymous sites; sil: silent sites; ${ }^{*} \mathrm{p} \leq 0.05$. 


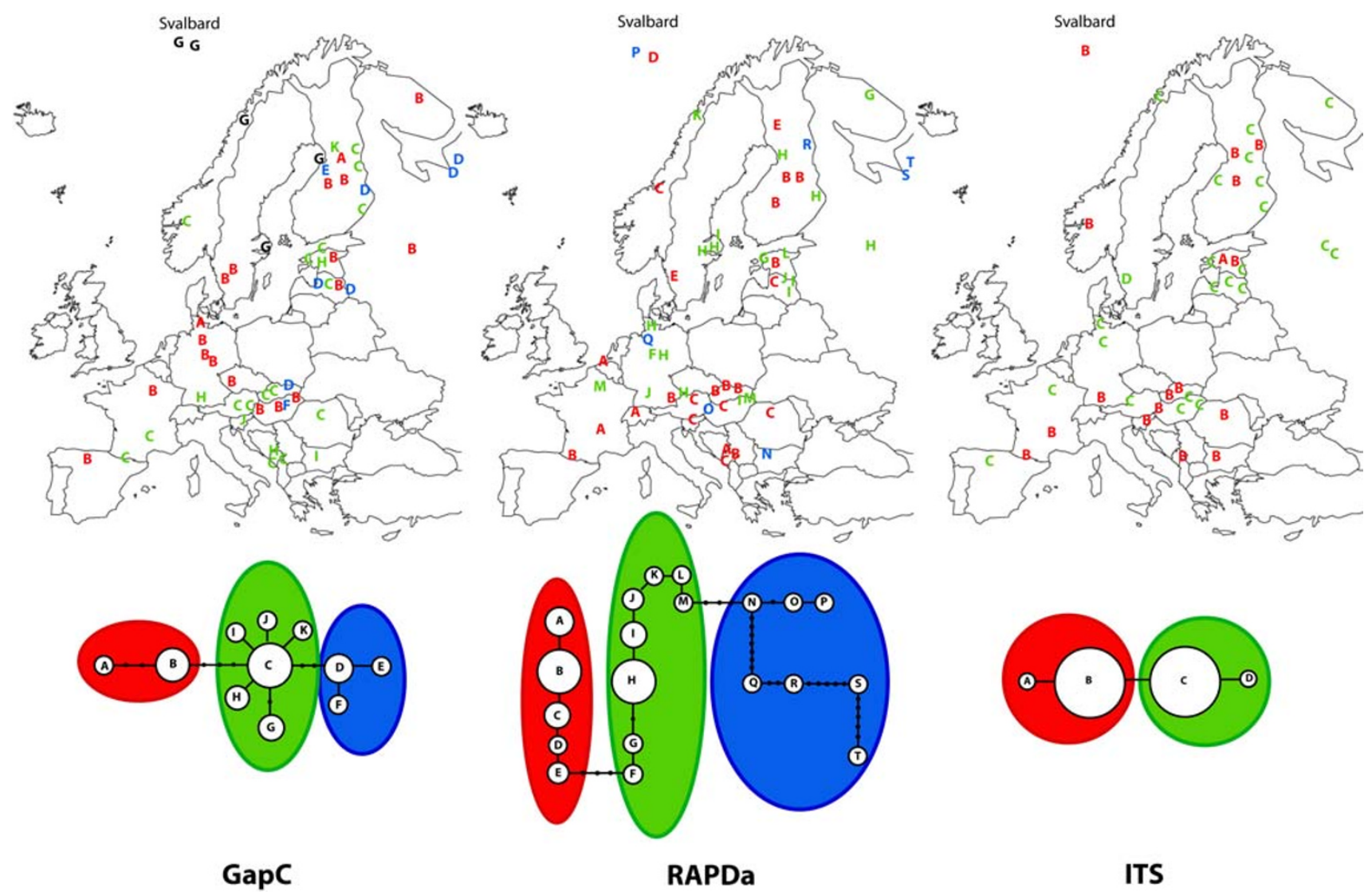

\section{Figure 2}

Geographic distribution of S. squarrosum haplotypes and the corresponding maximum parsimony networks. In each haplotype network, letters in circles represent haplotypes found. Missing haplotypes are marked with black dots and lines connecting haplotypes denote one mutational change. Well-differentiated clades are shown within red, green and blue ellipses. Letters on the map have the colour of the corresponding genetic clade.

rate of $S$. fimbriatum in the Non-Atlantic clade was significantly different from zero, whereas that of $S$. squarrosum might come from a declining, expanding or stable population as well. Although likelihood surfaces were relatively flat, growth rate values of the two species turned out to be significantly different taking approximate confidence intervals into account.

In the mismatch distribution analysis, only the GapC sequences were used because of the low variability of the RAPDa and ITS regions (Table 3 and 4). The sudden demographic and the spatial expansion model both fit mismatch data of $S$. squarrosum. However, the fit was better using the spatial expansion model. The mismatch distribution of all European accessions of $S$. fimbriatum was significantly different from the pure demographic expansion model, but matched the spatial expansion model. Tau was at least 4 times larger in $S$. squarrosum than in S. fimbria- tum. Theta estimated by the spatial expansion model was greater in S. fimbriatum, but this difference was not statistically significant. Estimation of parameters of sudden population growth or population expansion models failed for the Non-Atlantic group of $S$. fimbriatum, because the nonlinear least squares algorithm failed to converge in ARLEQUIN.

\section{Fitting the data to the IM model}

The provided population genetic parameter estimates of the IM model are all scaled with the mutation rate. In the absence of reliable mutation rate estimates of the regions results were interpreted in a relative way (Figure 3). Current theta values of the Atlantic and Non-Atlantic clades of $S$. fimbriatum showed considerably different distributions and point estimates. The Atlantic clade had lower theta than the Non-Atlantic clade and theta of the ancestral population was much lower than both of them. Estimate of 
Table 2: Test of population growth using $F_{u}$ 's $F_{s}$ statistic. Calculations were done including only nucleotide substitutions or nucleotide substitutions and gaps in the analysis.

\begin{tabular}{llll}
\hline & Fu's $\mathrm{F}_{\mathrm{s}}$ (with gaps/without gaps) & \\
\hline & Atlantic & Non-Atlantic \\
\cline { 2 - 4 } & & & \\
S. fimbriatum & & $-8.4558 * / 0.7145$ & Total data set \\
RAPDa & $-0.5938 /-0.3393$ & $-7.1366 * /-5.2437 *$ & $-8.0260 * / 0.7850$ \\
ITS & $-0.3393 / \mathrm{nc}$ & $-15.4390 * /-11.5356 *$ & $-6.3052 * /-1.7310$ \\
GapC & $0.5410 / 0.8564$ & - & $-10.7240 * /-7.4648 *$ \\
S. squarrosum & - & - & $-0.4350 /-1.7750$ \\
RAPDa & - & $-0.6240 / 1.2670$ \\
ITS & - & & \\
GapC & & &
\end{tabular}

theta for the Non-Atlantic clade was flat and converged but did not reach zero even after considerable extension of the prior. Scaled migration parameters were all very close to zero. Point estimate of the splitting parameter was high.

In contrast to $S$. fimbriatum, current thetas in S. squarrosum almost gave the same point estimates. Point estimate of the ancestral neutral mutation parameter was an order of magnitude higher than that of the current populations, but the posterior distribution was relatively flat. It converged to zero at higher theta values but did not reach zero even after extending the prior of theta considerably. Scaled migration parameters showed non zero values but were flat. Posterior probability distribution of the splitting parameter was flat and uninformative (not shown).

\section{Discussion}

Phylogeographic structure

Results of the present analysis confirm the divergence of an Atlantic and a Non-Atlantic lineage in S. fimbriatum and almost no geographic population structure in $S$. squarrosum [28]. Moreover, our current results evidently support a genome-wide historical event, which influenced all regions in the same way regardless of genomic position.
Since there is no reliable calibration point in the history of the genus, it is not possible to date the split in S. fimbriatum. In absence of dating, at least two hypotheses are plausible. The first one assumes that Quaternary cold periods were long enough to allow accumulation of the observed number of mutations among the Atlantic and Non-Atlantic groups of populations. In addition, it presumes that current geographic position of populations roughly correspond to that of the historical ones. The Atlantic group might have survived along the Atlantic coast of Spain, France and South England, but location of refugia of the Non-Atlantic group remains ambiguous. Results suggest that Eastern Iberian populations represent recent colonization events. Using reconstructions of Gajewski et al. [29] based on peat moss spores we hypothesize survival of the Non-Atlantic lineage along the border of Poland and Slovakia where extensive Sphagnum dominated habitats occurred in about 19 ky before present.

The second hypothesis assumes intercontinental migration between Europe and America. North American and European populations of several Sphagnum species appear to be closely related [30-33]. Among others, long-range dispersal as a plausible explanation for the observed pattern has been suggested [26,34,35]. Assuming effective intercontinental migration, colonization from a divergent

Table 3: Test of population growth using mismatch distribution analysis. Analysis was conducted using all European accessions of both species. Note that estimations are given only for the GapC gene.

\begin{tabular}{|c|c|c|c|c|c|c|c|}
\hline \multicolumn{8}{|c|}{ Mismatch analysis } \\
\hline & \multicolumn{4}{|c|}{ Pure demographic expansion } & \multicolumn{3}{|c|}{ Spatial expansion } \\
\hline & $P$ & Tau & $\theta_{0}$ & $\theta_{1}$ & $\mathrm{P}$ & Tau & $\theta_{0}$ \\
\hline S. fimbriatum & 0.026 & - & - & - & 0.710 & $0.325(0.210-4.710)$ & $0.782(0.000-1.652)$ \\
\hline S. squarrosum & 0.143 & $4.976(1.860-9.362)$ & $0.000(0.000-1.813)$ & $6.509(2.843-88.003)$ & 0.257 & 4.195 (1.678-7.446) & $0.092(0.000-1.995)$ \\
\hline
\end{tabular}

p: significance of fit (observed vs. expected distributions); Tau: scaled time elapsed since the expansion; $\theta_{0}$ : theta of the population before expansion; $\theta_{1}$ : theta of the population after expansion; $90 \%$ confidence intervals are given in brackets. 
Table 4: Test of population growth using maximum likelihood estimation. Analysis was conducted using all European accessions of both species. Note that estimations are given only for the GapC gene.

Maximum likelihood estimates

\begin{tabular}{|c|c|c|c|c|}
\hline & \multicolumn{2}{|l|}{ Non-Atlantic clade } & \multicolumn{2}{|l|}{ Europe } \\
\hline & $\theta$ & $g$ & $\theta$ & $g$ \\
\hline S. fimbriatum & $0.01218(0.00393-0.06008)$ & $15000.00(783|.62-325| 3.97)$ & $0.00377(0.00176-0.00678)$ & $857.04(-622.83-5891.93)$ \\
\hline S. squarrosum & - & - & $0.00242(0.00113-0.00563)$ & $233.58(-1244.40-2401.97)$ \\
\hline
\end{tabular}

$\theta$ : maximum likelihood estimator of the neutral mutation parameter; $\mathrm{g}$ : the exponential growth parameter; $95 \%$ percentile intervals of values around the most likely estimates are shown in brackets.

American population might account for the difference between the two lineages. To test this hypothesis we analysed additional samples from different parts of North America. Most of the North American accessions either shared haplotypes with the Non-Atlantic group or differed by only one substitution and represented derived tip haplotypes. Therefore recent colonization from America does not account for the divergence of the Atlantic and NonAtlantic clades.

In addition, several issues indicate that the Quaternary period has played an important role in the observed genetic structure of the species. First, the patterns detected in this study were very similar to those found in other bryophyte species [36-38] and seed plants $[39,40]$. The second reason comes from fossil data and climatic reconstructions. Occurrence of relic Sphagnum species [20], fossil remains of peat moss spores or leaves [41,42] and climatic reconstructions also support survival of populations along the Atlantic coast of Europe [29,43]. Consequently, our results and the above-mentioned observations support the separation of the Atlantic and Non-Atlantic lineages of S. fimbriatum by Quaternary glacial cycles and reject recent colonization from another continent as the cause of the split.

\section{Population demography}

Selection influences each gene differently, whereas demographic processes affect the individual as a whole and thus influence all parts of the genome uniformly regardless of genomic position [18]. Hence, in an expanding population an excess of low frequency variants is expected for all loci. This leads to negative Tajima's $D$ and Fu's $F_{s}$ statistics $[44,45]$. These statistics all analyse the total or relative number of singleton mutations compared to the neutral coalescent. Their power depends on the total number of segregating sites in the data set and the time elapsed since the demographic event $[45,46]$. Therefore it is not surprising that some of the statistics were not significant when excluding gaps as segregating sites from the analyses. This shows that substitution rates are relatively low compared to the time elapsed since the demographic event. Increas- ing the mutation rate with including gaps as mutations in the calculations improves power of the tests and extends the time frame in which those are applicable. Using these assumptions, all test statistics for all regions of $S$. fimbriatum were significantly different from the neutral expectations, whereas none of them provided significant deviations in S. squarrosum. This confirms a general excess of low frequency variants, in S. fimbriatum especially in the Non-Atlantic clade. Since the regions used are unlinked, we favour a population demographic expansion against a genome wide selection hypothesis in S. fimbriatum. Sequencing errors are negligible because both species were analysed using the same protocols and polymorphisms have been rechecked on the original chromatograms.

Mismatch distribution analysis revealed further details about the demographic history of the two species. Mismatch distribution of spatial expansion only slightly differs from that of pure demographic expansion, the latter showing a higher frequency of identical sequences [47]. In case of $S$. fimbriatum, data support the spatial expansion model, which is in concordance with the hypotheses about rapid spatial expansion of the species after the Quaternary glaciations. Data from S. squarrosum fit both scenarios, however, considerably better match the latter. This suggests that $S$. squarrosum probably went through spatial expansion as well. Tau value of $S$. squarrosum was approximately an order of magnitude higher than that of S. fimbriatum, although they were not significantly different. This suggests that expansion of $S$. fimbriatum is more recent than that of $S$. squarrosum. Assuming that $S$. fimbriatum expanded immediately after the Last Glacial Maximum (LGM), the estimate for $S$. squarrosum suggests expansion related to earlier geological or climatic events than the LGM. However, results of mismatch analyses need to be interpreted with caution. These analyses do not use genealogical information included in the data and testing the fit between observed and expected distributions and estimating approximate confidence intervals represent a complex problem as well. Estimation and confidence intervals of tau and $\theta$ are skewed and biased, espe- 


\section{Sphagnum fimbriatum}
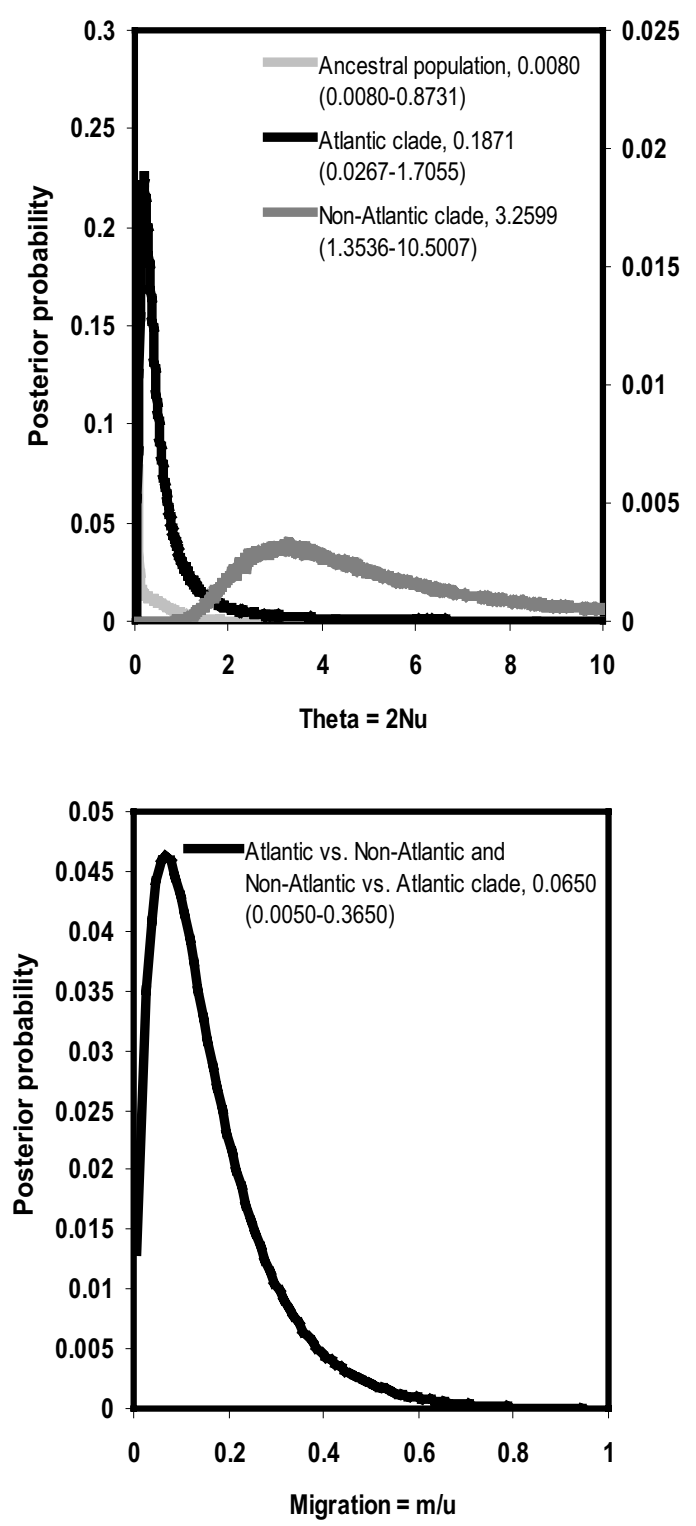

Sphagnum squarrosum
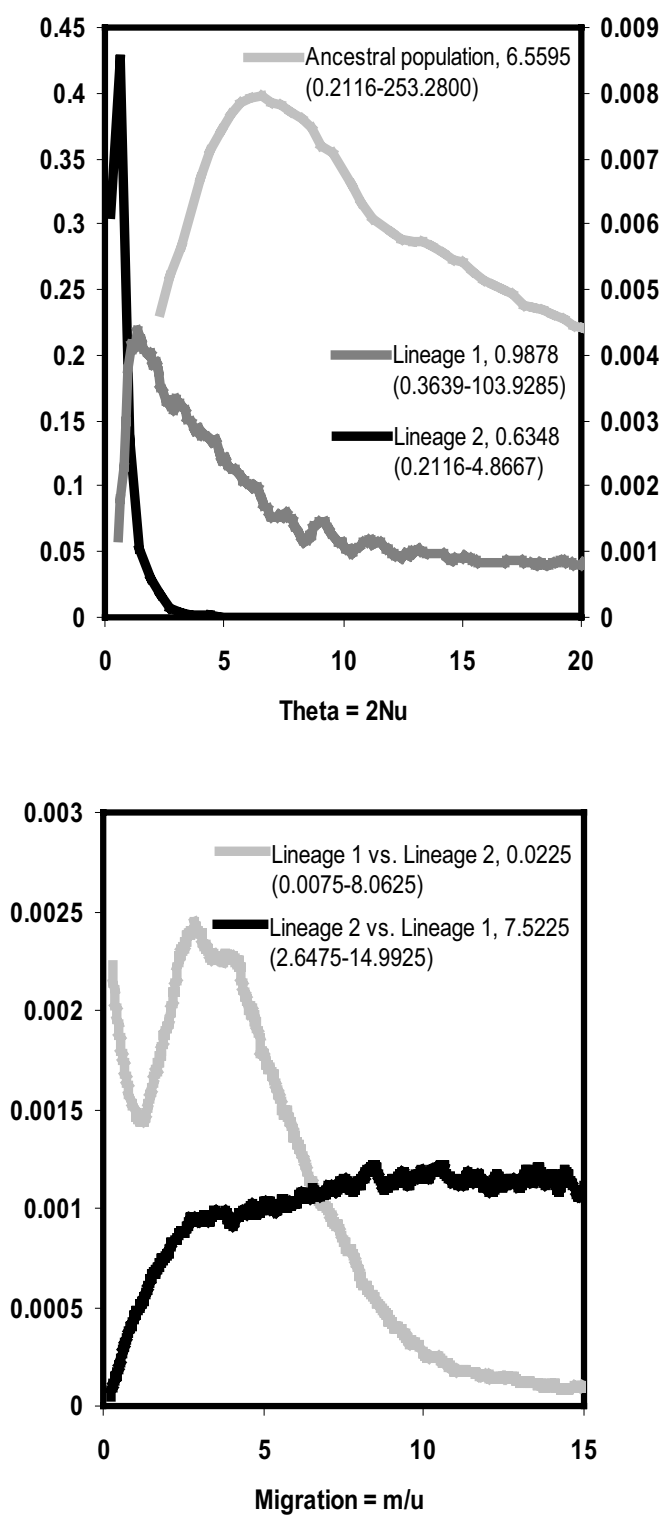

Figure 3

Posterior probability density functions of the parameters estimated using the Isolation with Migration model. In the figures point estimates of each parameter and the $90 \%$ highest posterior density intervals (in brackets) are given for each parameter. Note that parameter estimates are scaled with mutation rates. $\mathrm{N}$ : effective population size, $\mathrm{m}$ : rate of migration per gene per generation, u: mutation rate per gene per generation. 
cially in data sets with low resolution [48]. Maximum likelihood (LAMARC) and Bayesian analyses (IM) also supported rapid population expansion in S. fimbriatum. Results of both analyses showed no significant evidence for population growth in S. squarrosum. The IM analysis even supported a population decline since the separation of the two lineages of S. squarrosum.

Information on the nucleotide polymorphism of three independent regions gives the opportunity to reconstruct the historical demography of the two species in details. Populations of S. fimbriatum and S. squarrosum have been separated by the Quaternary glaciations, which led to the differentiation of two and three genetically well diverged European lineages, respectively. Fixed replacement mutations among the Atlantic and Non-Atlantic groups of $S$. fimbriatum indicate that both lineages went through a severe bottleneck, which led to the fixation of slightly deleterious mutations. It is worth noting that populations of the Atlantic group retained relatively divergent haplotypes (Figure 1) despite of their scattered occurrence and small extent (occurrences are usually not larger than $5 \times 5 \mathrm{~m}$ ). Hence, these populations very likely represent the leftovers of a formerly more widely distributed genetic lineage. Fitting the IM model to the data set suggests that a very high proportion of the ancient population of S. fimbriatum founded the Atlantic clade and the size of the ancient population was very small. These results show that the Atlantic clade is very likely a direct descendant of the ancestral population, which retained ancestral haplotypes and went through a severe bottleneck. IM and maximum likelihood estimations show that the Quaternary glaciations shaped population size of the two species differently. Ancient population size of $S$. squarrosum is considerably larger than that of $S$. fimbriatum indicating a more severe bottleneck in the latter. Larger effective population size of the ancient population of S. squarrosum also explains the lack of replacement mutations in coding regions as well as the lack of geographic structure of haplotypes.

\section{Pattern and amount of molecular variability}

Overall diversity values for the ITS region were usually an order of magnitude higher in $S$. fimbriatum than in $S$. squarrosum, however all values were very low. Moreover, in S. fimbriatum, a considerable portion of the diversity was due to the genetic differentiation between Atlantic and Non-Atlantic clades. In contrast, GapC sequences of the species showed nearly the same nucleotide diversity values. This is an unexpected result because the pioneer characteristic of $S$. fimbriatum (Sundberg et al. 2006), even when combined with a parallel reduction of the historical population size of $S$. squarrosum, implies lower level of molecular polymorphism in the first species. Peat moss populations are likely to function as a metapopulation
$[49,50]$. Theory shows that species-wide genetic diversity is rapidly lost in metapopulations of pioneer species if among population migration rates are lower than extinction/recolonization rates $[51,52]$. Although pioneer characteristic reduces the level of molecular diversity, in range expansion, due to the phenomenon of surfing mutations, molecular diversity is increased by a higher proportion of retained neutral mutations [53]. Consequently, it seems likely that range expansion contributed significantly to the molecular variability of $S$. fimbriatum.

Although the magnitude of molecular polymorphism was similar in both species, its pattern differed considerably showing an excess of polymorphism in coding regions of $S$. fimbriatum compared to $S$. squarrosum. Recent increase of effective population size results in a significant excess of slightly deleterious replacement mutations [54]. Weakened selection due to reduced effective population size can lead to the accumulation of slightly deleterious mutations as well and thus to elevated nucleotide diversity in selfing plants [55]. The McDonald-Kreitman test showed no significant deviations from neutral expectations for the GapC gene, however, exons of this gene contained replacement mutations in $S$. fimbriatum whereas in $S$. squarrosum all mutations were restricted to noncoding regions. Replacement mutations in S. fimbriatum (Lys-Arg, Ile-Val, Lys-Gln) caused no change in hydrophoby and almost no change in $\mathrm{pI}$ values and thus are likely to be only slightly deleterious. Consequently, elevated number of replacement mutations in S. fimbriatum also supports a rapid population demographic expansion in Europe.

Both species are easily dispersed by spores to longer distances $[26,27]$, however, nuclear markers resolved distinct Atlantic and Non-Atlantic clades only in S. fimbriatum. All markers indicate either restricted migration or establishment potential measured on the time scale of the markers' mutation rates. S. fimbriatum produces considerable amounts of spores, which can germinate on a wider range of substrates (especially on substrates with low phosphate availability) compared to other Sphagnum species $[26,56]$. Based on these characteristics of the species, the NonAtlantic clade of $S$. fimbriatum might have rapidly recolonized available soil surfaces after the last glaciation. In absence of further space, the Atlantic clade might have been unable to follow this lineage and remained restricted to the Atlantic coast of Europe. Similar patterns of leading edge colonization have been observed in several tree species in Europe [57,58].

Overall nucleotide diversity of the three regions was low in both species compared to estimations in seed plants [reviewed in [18]], which might be a general trend in bryophytes. Due to the haploid gametophores, no sheltering of recessive alleles exists in bryophytes and the haploid 
genome of each individual is directly exposed to selection $[30,59]$. This, coupled with the presence of deleterious mutations, considerably reduces the amount of genetic diversity. Mutation rate also influences genetic variability. Selection against high mutation rates should exist in bryophytes; otherwise the presence of slightly deleterious mutations will lead to a severe mutational load [60]. Our results show that slightly deleterious mutations are present but are removed very efficiently. The theory of selection directly acting on the gametophores and low mutation rates are supported by the data set presented here. Assuming the same mutation rates in both species, selection is so efficient, that no replacement mutations were found in the GapC gene of S. squarrosum at all. Interestingly, in a previous study McDaniel and Shaw [61] investigated the level of nucleotide polymorphism in the moss Ceratodon purpureus which turned out to be an order of magnitude higher than in our species. As this investigation relied on a worldwide sample and used multi-copy genes, high level of polymorphism may reflect the complex interplay of various forces, such as evolution of gene families, selective sweeps and phylogeographic history. Hence, the two studies are hardly comparable and further investigations on the extent of population level sequence polymorphism are needed to test theoretical predictions. In contrast to our results, high levels of isozyme diversity were found within populations of several bryophytes $[30,60]$. This has partly been interpreted as a result of local adaptation [61]. Although local adaptation might be important in several species, our results do not support this hypothesis, since only one of the three replacement mutations in the GapC gene resulted in charge change. Hence these mutations probably do not influence enzyme functions significantly.

\section{Conclusion}

Analysing nucleotide polymorphisms of multiple loci combined with the application of the coalescent theory provides an effective way to draw inferences about historical demography of species. Furthermore, investigating polymorphisms of several independent loci makes it possible to separate the effects of selective and demographic forces. Our study evidently shows that species with similar ecology, mating systems and current distributions may have very different demographic and phylogeographic histories. Such cryptic differences are probably frequent among bryophytes and must be taken into account when assessing the vulnerability of species and planning conservation strategies. Due to their haploid gametophores, bryophytes are especially appropriate for studies on sequence level polymorphisms. Future research using nucleotide polymorphism data will certainly discover undetected demographic, phylogeographic and evolutionary processes in this group of haploid plants.

\section{Methods \\ Sampling and DNA extraction}

S. fimbriatum and S. squarrosum plants were collected by the authors, provided by colleagues or obtained from herbaria [see Additional file 1]. Plants were dried and stored in silica gel at $4^{\circ} \mathrm{C}$. DNA was extracted using the DNAeasy plant mini kit (Qiagen, Switzerland) following the manufacturers protocol. Three regions of the nuclear genome were used for sequencing. The region spanning the 5th9'th exons of the GapC (glyceraldehyde-3-phosphatedehydrogenase C) gene (appr. 1500 bps) was amplified using protocols and primers described in Szövényi et al. [64]. A genomic region with unknown function [RAPDa sensu [65]] was also used. New primers were designed to improve amplification and sequencing of the fragment. As a third locus the ITS1-5.8S-ITS2 region (hereafter ITS) were amplified and sequenced. Yield of PCR reactions were improved by applying a semi-nested PCR, where $1 \mu \mathrm{l}$ of the $10 \times$ diluted first round product was used as template in the second reaction [see Additional file 2]. The PCR reaction contained the same amount of components as described in Szövényi et al. [64] except that final DMSO concentration was set to $1 \% \mathrm{v} / \mathrm{v}$ in the PCR mastermix to amplify the ITS region. PCR reactions were run on Biometra (T1 or Tgradient, Whatman Biometra) and Techne ptc 412 (Barloworld Scientific Ltd.) thermocyclers. The PCR program was the following: $94^{\circ} \mathrm{C}$ denaturation for $4 \mathrm{~min}$, then $94^{\circ} \mathrm{C}$ for $1 \mathrm{~min}$, different annealing temperatures for $1 \mathrm{~min}$ and $72^{\circ} \mathrm{C}$ extension for $1-1.5 \mathrm{~min}$ [see Additional file 2] with 35 cycles with a final extension step at $72{ }^{\circ} \mathrm{C}$ for $7 \mathrm{~min}$. The products were checked on $0.8 \%$ agarose gels and cleaned using the GFX PCR and gel band purification kit (Amersham Biosciences, Switzerland). Approximately $10 \mathrm{ng}$ product was sequenced in $10 \mu \mathrm{l}$ cycle sequencing reaction with the Big dye v3.1 in an ABI prism 3100 (Applied Biosystems) genetic analyzer using either the original and/or different internal PCR primers [see Additional file 2]. Sequences were contiged and corrected if necessary with the Sequencher 4.5 software (Gene Code Corporation).

\section{Sequence alignment, molecular diversity and recombination event estimates}

Sequences were aligned using ClustalW [66], checked by eye and adjusted if needed. All polymorphic sites were rechecked on the original electropherograms and corrected to avoid false base callings. Haplotype networks were reconstructed using the TCS software [67]. To describe the infra specific molecular diversity of the regions Watterson's $\left(\theta_{\mathrm{w}}\right)$ [68] and Tajima's $\left(\theta_{\pi}\right)$ [44] moment estimators of the neutral mutation parameter were calculated. In case of the GapC gene, levels of polymorphism were calculated for synonymous, nonsynonymous and silent sites as well. 
All regions were tested for recombination using the four gamete test and the minimum number of recombination events during the history of the sample was calculated $\left[R_{m},[69]\right]$. For several following analyses (cf. below) sequences with incompatible nucleotide pairs were cut into recombination free blocks. All calculations were done using dnaSP v.4.10.4 [70].

\section{Testing deviation from neutrality}

To test whether the sequenced regions evolved under neutral expectations, four different methods were applied. The multilocus HKA test was conducted using the HKA software [71]. 10000 coalescent simulations were run to generate the null distribution of $\chi^{2}$ values. Neutral evolution of the GapC gene was also tested with the McDonaldKreitman test [72]. To test the neutraliy of each locus separately, Tajima's D [44] and Fay and Wu's H statistics [73] were calculated for each of them. For each locus the latter two test statistics were calculated considering nucleotide substitutions alone and recoding gaps as well. Gaps were coded as single and independent events. Due to the low variability and non-overlapping occurrence of gaps in the data set, alignment, assessment of homology and subsequent coding of gaps was straightforward.

\section{Analysis of population growth}

As a powerful descriptive statistic of population growth, Fu's $F_{s}[74]$, was calculated using frequency distribution of alleles for each region separately. Fu's $\mathrm{F}_{\mathrm{s}}$ statistic compares the number of alleles expected under the neutral coalescent in a constant sized population based on the neutral mutation parameter and the size of the sample. In an expanding population large negative values are expected due to the excess of rare mutations. Populations after a demographic expansion show a star-like phylogeny and a unimodal mismatch distribution [15], where the peak corresponds to the time of the expansion. The method of Schneider and Excoffier [48] implemented in ARLEQUIN 3.0 [75] was used to fit observed and expected mismatch distributions and to estimate parameters of a pure demographic and a spatial expansion model. The two models are expected to produce different mismatch distributions when the number of migrants between populations is not large. Otherwise the two hypotheses can not be distinguished. In this study both models were considered to complement genealogical methods that fail to detect signs of a spatial expansion.

As an alternative, maximum likelihood estimation of the exponential growth rate and historical $\theta$ was conducted. The software LAMARC 2.0 [11] was used to get estimates of growth rates for all European accessions of $S$. squarrosum and $S$. fimbriatum and for the Non-Atlantic clade of $S$. fimbriatum. 30 short chains (20 000 steps each) and 2 long chains (200 000 steps) with a sampling interval of 20 steps and a burn in period of 1000 and 10000 respectively were applied.

The IM software $[76,77]$ was used to fit the data to the Isolation with Migration model (IM). This model assumes two populations connected by migration, which are derived by the splitting of an ancient population. The IM model estimates several parameters simultaneously and therefore describes processes in natural populations in their complexity. Populations were defined based on our a priory knowledge of chloroplast lineages using the parsimony networks of each species [28]. Runs were conducted for the combined dataset including all three nuclear regions sequenced. Initial runs with wide priors were used to delimit the plausible range of priors. Since indels of all three regions were informative, analyses have been conducted both with and without indels coded. Including gaps as additional characters did not change the multilocus parameter estimates but narrowed their confidence intervals, however, only results without coding gaps are shown. The infinite site mutation model of sequence evolution was applied and loci were cut into non-recombining blocks. In case of the RAPDa sequences of S. squarrosum, only the microsatellite region was used. Each run was introduced by a 100000 steps long burn-in period. Multilocus analyses were run using 5-7 parallel chains under a linear heating scheme with a heating value of 0.05 - 0.005. Convergence of parameters and mixing of chains were followed by visual inspection of parameter trend lines and checking of ESS values. Analyses were run until the lowest ESS value reached minimum 200. In all analyses population demographic changes were allowed.

\section{Authors' contributions}

PS and ZH initiated the study, carried out the molecular genetic studies, analysed the data and wrote the manuscript draft. ZT intensively helped in collecting. JJS and ZT supervised the work, contributed to the analyses of the results and to the writing of the paper. All authors read and improved the final manuscript.

\section{Additional material}

\footnotetext{
Additional file 1

Collection data and GenBank numbers of accessions analysed. Detailed list of the Sphagnum fimbriatum and S. squarrosum accessions analysed for the three regions in this study. Letters refer to haplotypes of Figure 1 and 2; na: not analysed.

Click here for file

[http://www.biomedcentral.com/content/supplementary/14712148-7-144-S1.pdf]
} 


\section{Additional file 2}

Primers used in this study. Names of newly designed primers are in bold. 1st and 2 nd refer to the first and the second round of a semi nested PCR. In the second round PCR $1 \mu \mathrm{l} 10 \times$ diluted first round product was used as template.

Click here for file

[http://www.biomedcentral.com/content/supplementary/14712148-7-144-S2.pdf]

\section{Acknowledgements}

We thank Liudmila Abramova, Baiba Bambe, Sam Bosanquet, Jürgen Brandt, Philippe de Zuttere, Christian Dolnik, Andrea Fodor, Anna Ganeva, Irina Goldberg, Pierre Goubet, Alessia Guggisberg, Patxi Heras, Heike Hofmann, Adam Hölzer, Michael Ignatov, Luca Miserere, Zoltán Illyés, Marta Infante, Nele Ingerpuu, Beatriz Itten, Monika Koperski, Robert Krisai, Ildikó László, Andrej Martinčič, Péter Ódor, Beáta Papp, Claes Persson, Ron Porley, Markus Preussing, José Pujos, Sigurd Såstad, Ana Seneca, Blanka and Jonathan Shaw, Rudolf Soltés, Sture Westerberg, Sebastian Sundberg, Erzsébet Szurdoki, Zoltán Tóth, Edwin Urmi, Alain Vanderpoorten, Kai Vellak, Risto Virtanen and Harald Zechmeister for collecting material and/ or for field assistance. Critical comments of two anonymous reviewers on an earlier version of the manuscript are greatly acknowledged. This research was financially supported by a Young Academics Grant of the University of Zurich (Forschungskredit der Universität Zürich), by a Travel Grant of the Swiss National Academy of Science and by a grant of the Hungarian Scientific Research Found (OTKA, NI 682I8).

\section{References}

I. Hewitt GM: Post-glacial re-colonization of European biota. Biol J Linn Soc 1999, 68:87-I I 2.

2. Hewitt GM: The genetic legacy of the Quaternary ice ages. Nature 2000, 405:907-913.

3. Fraser SM, Lawton JH: Host range expansion by British moths onto introduced conifers. Ecol Entomol 1994, 19:127-137.

4. Losos JB: Dynamics of range expansion by three introduced species of Anolis lizards on Bermuda. J Herpetol 1996, 30:204-210.

5. Sakai AK, Allendorf FW, Holt JS, Lodge DM, Molofsky J, With KA, Baughman S, Cabin RJ, Cohen JE, Ellstrand NC, McCauley DE, O'Neil P, Parker IM, Thompson JN, Weller SG: The population biology of invasive species. Annu Rev Ecol Syst 200I, 32:305-332.

6. Radosevich SR, Stubbs MM, Ghersa CM: Plant invasions--process and patterns. Weed Sci 2003, 5I:254-259.

7. Weber E: Current and potential ranges of three exotic goldenrods (Solidago) in Europe. Conserv Biol 200I, 15:122-I28.

8. Hedenäs L, Bisang I, Tehler A, Hamnede M, Jaederfelt K, Odelvik G: A herbarium-based method for estimates of temporal frequency changes: mosses in Sweden. Biol Conserv 2002, 105:32I-33I.

9. Beaumont MA: Detecting population expansion and decline using microsatellites. Genetics 1999, 153:2013-2029.

10. Storz JF, Beaumont MA, Alberts SC: Genetic evidence for longterm population decline in a savannah-dwelling primate: inferences from a hierarchical Bayesian model. Mol. Biol. Evol 2002, 19:198I-1990

II. Kuhner MK, Yamato ], Beerli P, et al.: LAMARC v 2.0. 2005 [http:/ levolution.gs.washington.edu/lamarc.html]. University of Washington

12. Goossens B, Chikhi L, Ancrenaz M, Lackman-Ancrenaz I, Anaud P, Bruford MW: Genetic signature of anthropogenic population collapse in orangutans. PLoS Biology 2006, 4:e25. doi:10.1371/journal.pbio.0040025

13. Rosenberg NA, Nordborg M: Genealogical trees, coalescent theory and the analysis of genetic polymorphisms. Nat Rev Genet 2002, 3:380-390.

14. Hein J, Schierup MH, Wiuf C: Gene Genealogies, Variation and Evolution A Primer in Coalescent Theory Oxford: Oxford University Press; 2004.
15. Slatkin M, Hudson RR: Pairwise comparisons of mitochondrial DNA sequences in stable and exponentially growing populations. Genetics 1991, I 29:555-562.

16. Rodgers AR, Harpending H: Population growth makes waves in the distribution of pairwise genetic differences. Mol. Biol. Evol 1992, 9(3):552-569.

17. Maynard Smith J, Haigh J: The hitchhiking effect of a favourable gene. Genet Res Camb 1974, 219:I I I4-III6.

18. Wright SI, Gaut BS: Molecular population genetics and the search for adaptive evolution in plants. Mol. Biol. Evol 2004, 22:506-519.

19. Nielsen R: Molecular signatures of natural selection. Annu Rev Genet 2005, 39:197-218.

20. Daniels RE, Eddy A: Handbook of European Sphagna Huntingdon: Institute of Terrestrial Ecology; 1985.

21. Cronberg N: Clonal structure and fertility in a sympatric population of the peat mosses Sphagnum rubellum and Sphagnum capillifolium. Can J Bot 1996, 74: / 375-I385.

22. Cronberg N, Natcheva R: Hybridization between the peat mosses, Sphagnum capillifolium and S. quinquefarium (Sphagnaceae, Bryophyta) as inferred by morphological characters and isozyme markers. Plant Syst Evol 2002, 234:53-70.

23. Shaw AJ, Melosik I, Cox C], Boles SB: Divergent and reticulate evolution in closely related species of Sphagnum section Subsecunda. Bryologist 2005, 108:363-376.

24. Flatberg KI, Thingsgaard K, Såstad SM: Interploidal gene flow and introgression in bryophytes. Sphagnum girgensohnii $\times$ S. russowii, a case of spontaneous neotriploidy. J Bryol 2006, 28:27-37.

25. Smith AJE: The Moss Flora of Britain and Ireland Second edition. Cambridge: Cambridge Univeristy Press; 2004.

26. Sundberg S, Hansson J, Rydin H: Colonisation of Sphagnum on land uplift islands in the Baltic Sea: time, area, distance and life history. | Biogeogr 2006, 33:|479-|49|.

27. Szurdoki E, Odor P: Distribution and expansion of Sphagnum fimbriatum Wils. in Hungary. Lindbergia 2005, 29: $136-142$.

28. Szövényi P, Hock Zs, Urmi E, Schneller J]: Contrasting phylogeographic patterns in Sphagnum fimbriatum and $S$. squarrosum (Bryophyta, Sphagnopsida) in Europe. New Phytol 2006, 172:784-794.

29. Gajewski K, Viau A, Sawada M, Atkinson D, Wilson S: Sphagnum peatland distribution in North America and Eurasia during the past 2I,000 years. Global Biogeochem Cyc 200I, I 5:297-3I0.

30. Wyatt R, Odrzykoski I, Stoneburner J: Isozyme evidence of reticulate evolution in mosses: Plagiomnium medium is anallopolyploid of $\boldsymbol{P}$. ellipticum $\times$ P. insigne. Syst Bot 1992, I 7:532-550.

31. Shaw AJ, Schneider RE: Genetic biogeography of the rare „,copper moss" Mielichhoferia elongata (Bryaceae). Am J Bot 1995, 82:8-17.

32. Såstad SM, Flatberg IK, Hanssen L: Origin, taxonomy and population structure of the allopolyploid peat moss Sphagnum majus. Plant Syst Evol 2000, 225:73-84.

33. Shaw AJ, Werner O, Ros RM: Intercontinental mediterranean disjunct mosses: morphological and molecular patterns. Am $J$ Bot 2003, 90:540-550.

34. Van Zanten BO: Experimental studies on trans-oceanic longrange dispersal of moss spores in the southern hemisphere. J Hattori Bot Lab 1978, 44:455-482.

35. Cronberg N: Colonization dynamics of the clonal moss Hylocomium splendens on islands in a Baltic land uplift area: reproduction, genet distribution and genetic variation. J Ecol 2002, 90:925-935.

36. Cronberg N: Population structure and interspecific differentiation of the peat moss sister species Sphagnum rubellum and S. capillifolium (Sphagnaceae) in northern Europe. Plant Syst and Evol 1998, 209:139-158.

37. Van der Velde M, Bijlsma R: Phylogeography of five Polytrichum species within Europe. Biol J Linn Soc 2003, 78:203-2I3.

38. Hedderson TA, Nowell TL: Phylogeography of Homalothecium sericeum (Hedw.) Br Eur; toward a reconstruction of glacial survival and postglacial migration. J Bryol 2006, 28:283-292.

39. Rendell S, Ennos RA: Chloroplast diversity in Calluna vulgaris (heather) populations in Europe. Mol Ecol 2002, I I:69-78.

40. Hofmann MH, Glass AS, Tomiuk J, Schmuths H, Fritsch RM, Bachman $\mathrm{K}$ : Analysis of molecular data of Arabidopsis thaliana (L.) 
Heyn. (Brassicaceae) with geographical information systems (GIS). Mol Ecol 2003, I 2:1007-1019.

4l. Forment $P$, Jovet-Ast S: Sphagnum imbricatum Russ., subfossil en France dans une tourbe des environs de Calais. Rev bryol lichénol 1950, 19:125-126.

42. Infante M, Heras P: Sphagnum imbricatum Hornsch. ex Russ., semifosil, en la turbera de Saldropo-Brazar (Ceanuri, Vizcaya). Est Inst Alavés Nat 1987, 2:201-203.

43. VanAndel TH, Tzedakis PC: Paleolithic landscapes of Europe and environs, I 50,000-25,000 years ago: an overview. Quaternary Sci Rev 1996, I5:48I-500.

44. Tajima F: Statistical method for testing the neutral mutation hypothesis by DNA polymorphism. Genetics 1989, I 23:585-595.

45. Ramos-Onsins SE, Rosas J: Statistical properties of new neutrality tests against population growth. Mol Biol Evol 2002 19:2092-2100

46. Sano A, Tachida H: Gene genealogy and properties of test statistics of neutrality under population growth. Genetics 2005, 169:1687-1697.

47. Excoffier L: Patterns of DNA sequence diversity and genetic structure after a range expansion: lessons from the infiniteisland model. Mol Ecol 2004, 1 3:853-864.

48. Schneider S, Excoffier L. Estimation of past demographic parameters from the distribution of pairwise differences when the mutation rates vary among sites: application to human mitochondrial DNA. Genetics 1999, 152:1079-1089.

49. Söderström L, Herben T: Dynamics of bryophyte metapopulations. In Advances in Bryology Volume 6. Edited by: Longton RE. Stuttgart: Science Publishers; 1997:205-24I.

50. Wakeley J, Alicar N: Gene genealogies in a metapopulation. Genetics 200I, 159:893-905

5I. Panell JR, Charlesworth B: Neutral genetic diversity in a metapopulation with recurrent local extinction and recolonization. Evolution 200I, 53:664-676.

52. Ingvarsson PK: A metapopulation perspective on genetic diversity and differentiation in partially self-fertilizing plants. Evolution 2002, 56:2368-2373.

53. Klopfstein $S$, Currat M, Excoffier $L$ : The fate of mutations surfing on the wave of a range expansion. Mol Biol Evol 2006, 23:482-490

54. Eyre-Walker A: Changing effective population size and the McDonald-Kreitman test. Genetics 2002, 162:2017-2024.

55. Charlesworth D, Wright SI: Breeding system and genome evolution. Curr Opin Genet Dev 200I, I I:685-690.

56. Sundberg S, Rydin H: Habitat requirements for establishment of Sphagnum from spores. J Ecol 2002, 90:268-278.

57. Demesure B, Comps B, Petit RJ: Chloroplast DNA phylogeography of the common beech (Fagus sylvatica $L$ ) in Europe. Evolution 1995, 50:2515-2520.

58. Heuertz M, Fineschi S, Anzidei M, Pastorelli R, Salvini D, Paule L, Frascaria-Lacoste N, Hardy OJ, Vekemans X, Vendramin GG: Chloroplast DNA variation and postglacial recolonization of common ash (Fraxinus excelsior L.) in Europe. Mol Ecol 2004, 13:3437-3452

59. Wyatt R, Stoneburner A, Odrzykoski IJ: Bryophyte isozymes: systematic and evolutionary implications. In Isozymes in Plant Bioogy Edited by: Soltis DE, Soltis P. Portland: Dioscorides Press; 1989:221-240.

60. Stenøien HK, Såstad SM: Genetic variability in bryophytes: does mating system really matter? J Bryol 200I, 23:313-319.

61. McDaniel SF, Shaw A]: Selective sweeps and intercontinental migration in the cosmopolitan moss Ceratodon purpureus (Hedw) Brid. Mol Ecol 2005, 14: I I2I-I I32.

62. Stenøien HK, Såstad SM: Genetic structure in three haploid peat mosses (Sphagnum). Heredity 1999, 82:391-400.

63. Shaw AJ: Population ecology, population genetics, and microevolution. In Bryophyte Biology Edited by: Shaw AJ, Goffinet B. Cambridge: Cambridge University Press; 2000:369-403.

64. Szövényi P, Hock Zs, Urmi E, Schneller J]: New primers for amplifying the GapC gene of bryophytes and its utility in infraspecific phylogenies in the genus Sphagnum. Lindbergia 2006, 31:32-39.

65. Shaw $A$, Cox $C$, Boles SB: Polarity of peatmoss evolution: who says mosses have no roots? Am J Bot 2003, 90:1777-1787.

66. Thompson JD, Higgins DG, Gibson T): CLUSTAL W: Improving the sensitivity of progressive multiple sequence alignment through sequence weighting, position-specific gap penalties and weight matrix choice. Nucleic Acids Res 1994, 22:4673-4680.

67. Clement M, Posada D, Crandall K: TCS: a computer program to estimate gene genealogies. Mol Ecol 2000, 9:1657-1660.

68. Hudson RR: Gene genealogies and the coalescent process. In Oxford Surveys in Evolutionary Biology Volume 7. Edited by: Harvey PH, Partridge L. New York: Oxford University Press; 1990: I-44.

69. Hudson RR, Kaplan NL: Statistical properties of the number of recombination events in the history of a sample of DNA sequences. Genetics 1985, I I I: I47-164.

70. Rozas J, Sanches-Delbarrio JC, Messeguer X, Rozas R: DnaSP, DNA polymorphism analyses by the coalescent and other methods. Bioinformatics 2003, 19:2496-2497.

7I. HKA - a program for conducting the HKA test of natural selection [http://lifesci.rutgers.edu/ heylab/HeylabSoft ware.htm\#HKA]

72. McDonald JH, Kreitman M: Adaptive protein evolution at the ADH locus in Drosophila. Nature 1991, 35 I:652-654.

73. Fay JC, $\mathrm{Wu} \mathrm{Cl}$ : Hitchhiking under positive Darwinian selection. Genetics 2000, 155: | 405-1413.

74. Fu YX: Statistical tests of neutrality of mutations against population growth, hitchhiking and background selection. Genetics 1997, 147:9|5-925.

75. Excoffier L, Laval G, Schneider S: Arlequin ver. 3.0: an integrated software package for population genetics data analysis. Evol Bioinf 2005, I :47-50.

76. Hey J, Nielsen R: Multilocus methods for estimating population sizes, migration rates and divergence time, with applications to the divergence of Drosophila pseudoobscura and D. persimilis. Genetics 2004, 167:747-760.

77. Hey J: On the number of new world founders: a population genetic portrait of the peopling of the Americas. PLOS Biol 2005, 3:el93.

Publish with Biomed Central and every scientist can read your work free of charge

"BioMed Central will be the most significant development for disseminating the results of biomedical research in our lifetime. "

Sir Paul Nurse, Cancer Research UK

Your research papers will be:

- available free of charge to the entire biomedical community

- peer reviewed and published immediately upon acceptance

- cited in PubMed and archived on PubMed Central

- yours - you keep the copyright
BiolMedcentral 